\title{
MODERNIZAÇÃO DO SISTEMA DE SUPERVISÃO DA MINA, BRITAGEM E CONCENTRADOR I DA SAMARCO MINERAÇÃO*
}

\author{
Alexandre Carvalho Martins ${ }^{1}$ \\ Eduardo Montenegro ${ }^{2}$ \\ Lucas Andery Reis ${ }^{3}$ \\ Luiz Henrique Silveira Moreira ${ }^{4}$ \\ Pablo Parreiras Drumond Ferreira ${ }^{5}$ \\ Rafael de Freitas Bachare ${ }^{6}$ \\ Vinícius Vilela Wiermann ${ }^{7}$
}

\section{Resumo}

Este trabalho tem por objetivo apresentar o projeto de modernização do sistema de supervisão da Mina, Britagem e Concentrador I da unidade de Germano da Samarco Mineração. A principal motivação que levou a Samarco a modernização do sistema de supervisão foi o fato da aplicação atual já estar saturada, dificultando novas implementações, além de já apresentar limitações no diagnóstico de defeitos e intertravamentos. Na solução implementada, destaca-se a estratégia de virtualização adotada, o sistema para cadastramento e modificação em tempo real das entidades do CLP e as funcionalidades para gestão de alarmes.

Palavras-chave: Sistema de supervisão; Virtualização; Gestão de alarmes; Samarco.

\section{MODERNIZATION OF THE SUPERVISORY SYSTEM OF THE MINE, CRUSHING PLANT AND CONCENTRATOR I OF SAMARCO}

\section{Abstract}

This work aims to present the control system modernization project of the Mine, Crushing Plant, and Mill plant I at Samarco Germano Unit. The main motivation for the project was the fact that the current application was already saturated, hindering new implementations, in addition to already presenting deficiencies in diagnosing defects and interlocks. In the implemented solution, it is highlighted the virtualization strategy adopted, the real time system for registration and modification of the PLC entities and the alarm management functionalities.

Keywords: Supervisory system; Virtualization; Alarm management; Samarco.

1 Bacharel em Engenharia da Computação, Engenheiro de automação, Gerência de Engenharia de Processo e Automação, Samarco, Mariana, Minas Gerais, Brasil.

2 Bacharel em Engenharia de controle e automação, Engenheiro de automação, IHM Engenharia, Belo Horizonte, Minas Gerais, Brasil.

3 Graduando em Engenharia de Controle e Automação, Técnico de automação, Gerência de Engenharia de Processo e Automação, Samarco, Mariana, Minas Gerais, Brasil.

4 Graduando em Engenharia da Computação, IHM Engenharia, Belo Horizonte, Minas Gerais, Brasil.

5 Bacharel em Engenharia de controle e automação, Engenheiro de automação, IHM Engenharia, Belo Horizonte, Minas Gerais, Brasil.

6 Bacharel em Engenharia de controle e automação, Engenheiro de automação, IHM Engenharia, Belo Horizonte, Minas Gerais, Brasil.

7 Bacharel em Engenharia Elétrica, Especialista em Automação Industrial, Engenheiro de automação especialista, Gerência de Engenharia de Processo e Automação, Samarco, Mariana, Minas Gerais, Brasil.

* Contribuição técnica ao $18^{\circ}$ Seminário de Automação e TI Industrial, 23 a 26 de setembro de 2014, São Paulo, SP, Brasil. 


\section{INTRODUÇÃO}

Este trabalho tem por objetivo apresentar o projeto de modernização do sistema de supervisão da unidade de Germano da Samarco Mineração. Mais especificamente das áreas da Mina, Britagem e Concentrador I.

Fundada em 1977, a Samarco é uma empresa brasileira de mineração, de capital fechado, controlada em partes iguais por dois acionistas: BHP Billiton Brasil Ltda. e Vale S.A. Seu principal produto é pelota de minério de ferro, produzida a partir da transformação de minerais de baixo teor em um produto nobre, de alto valor agregado, e comercializado para a indústria siderúrgica mundial. A Samarco é essencialmente uma empresa exportadora e, em 2012, comercializou sua produção para siderúrgicas de 25 países das Américas, Ásia, África, Europa e Oriente Médio.

\section{MOTIVAÇÃO E PREMISSAS DO PROJETO}

A principal motivação que levou a Samarco a modernização do sistema de supervisão foi o fato da aplicação atual já estar saturada, dificultando novas implementações, além de já apresentar limitações no diagnóstico de defeitos e intertravamentos. Neste sentido, ficou premente a necessidade de se implantar um novo sistema de supervisão que fosse capaz de superar estas dificuldades e implementar novas funcionalidades ao sistema.

O projeto de modernização consistiu na troca do sistema de supervisão e reprogramação de alguns CLPs. Abrange basicamente três áreas: Mina, Britagem e Concentrador I (Concentração, Reagentes e Utilidades) da Samarco Mineração, localizadas na Unidade Germano (Mariana - MG). O novo sistema de supervisão substitui os sistemas existentes desenvolvidos na plataforma Wonderware InTouch 7.0 e InTouch7.11.

Dentre as premissas exigidas pela Samarco para o desenvolvimento do sistema, podemos destacar:

- Manter a interface com sistemas de nível hierarquicamente superior (Gerenciamento da Produção - PIMS/MES) e sistemas legados (Sistema Analisador de Teor de Minério On-Line, Sistema de Análise de Tamanho de Partículas, Analisadores de Sílica e Flotação e Sistema de Controle Otimizante);

- Ser modular e flexível, permitindo expansão do sistema, tanto em número de clientes, quanto de servidores, PLCs e número de entidades (equipamentos, analógicas, PIDs, etc);

- Possibilitar cada estação de operação operar a sua área de aplicação, além de visualizar, completamente, as outras duas áreas de processo;

- Realizar a migração dos aplicativos com as respectivas plantas em operação;

- Tempo de navegação entre telas de processo (com 100\% das animações e tags carregadas): até 1,5 segundo;

- Tempo de abertura de janela pop-up de operação (Janela de equipamento, Analógica e PID) com 100\% das animações, objetos e tags associadas carregadas: até 1 segundo;

- Tempo de abertura de telas de cadastro com 100\% dos objetos, animações e tags associadas carregadas: até 3 segundos;

- Tempo de consulta a base histórica de alarmes e eventos com 1000 registros: até 3 segundos;

* Contribuição técnica ao $18^{\circ}$ Seminário de Automação e TI Industrial, 23 a 26 de setembro de 2014, São Paulo, SP, Brasil. 
- O sistema deve ser capaz de executar a lógica de mascaramento de estado, defeito ou intertravamento de 1000 equipamentos em até 1 segundo;

- Tempo de chaveamento de redundância entre servidores: até 15 segundos;

- Sistema deve funcionar em regime contínuo com 100\% de disponibilidade;

- Disponibilização de dados históricos (variáveis de processo, alarme e eventos) para consulta pelos usuários do sistema por um período de 6 meses;

\section{DETALHAMENTO DO PROJETO}

\subsection{Arquitetura do Sistema}

Na figura a seguir é apresentado a arquitetura funcional do sistema.



Figura 1. Arquitetura funcional do sistema.

\subsubsection{Plataforma de hardware}

O hardware desta solução virtualizada está dividido em 2 sites. Sendo o primeiro site responsável pelas aplicações principais da Mina, Britagem e Concentrador I, e o segundo site responsável por rodar as aplicações redundantes da solução. Dois storages físicos, em regime de sincronização contínua, armazenam os discos virtuais e toda a base de dados (Histórico e de cadastro) da aplicação.

Segue abaixo a descrição detalhada de toda a plataforma de Hardware usada na solução.

* Contribuição técnica ao $18^{\circ}$ Seminário de Automação e TI Industrial, 23 a 26 de setembro de 2014, São Paulo, SP, Brasil. 
Tabela 1. Plataforma de hardware, funções e aplicativos

\begin{tabular}{|c|c|c|c|}
\hline $\begin{array}{l}\text { Tipo de } \\
\text { Servidor }\end{array}$ & SITE & Funções & Aplicativos a executar \\
\hline $\begin{array}{l}\text { Blade } \\
\text { M610 }\end{array}$ & $\begin{array}{l}\text { CONCENTRADOR } \\
1\end{array}$ & $\begin{array}{l}\text { Servidor de aplicação } \\
\text { principal SCADA (Mina, } \\
\text { Britagem e } \\
\text { Concentrador) }\end{array}$ & $\begin{array}{ll}\text { - } & \text { E3 Server } \\
\text { - } & \text { EPM Interface } \\
\text { - } & \text { E3 Dominios remotes } \\
\text { - } & \text { E3 OPC Server } \\
\text { - } & \text { Driver Ge-Fanuc } \\
& \text { ethernet } \\
\text { - } & \text { Driver Allen Bradley } \\
& \text { ABFD1 }\end{array}$ \\
\hline $\begin{array}{l}\text { Blade } \\
\text { M610 }\end{array}$ & $\begin{array}{l}\text { CONCENTRADOR } \\
1\end{array}$ & $\begin{array}{l}\text { Servidor de Historiação } \\
+ \text { BD }\end{array}$ & $\begin{array}{ll}\text { - } & \text { EPM Server } \\
\text { - } & \text { SQL Server } 2008 \\
\text { - } & \text { E3 Web Server } \\
\end{array}$ \\
\hline $\begin{array}{l}\text { Storage } \\
\text { PS } \\
4100 x\end{array}$ & $\begin{array}{l}\text { CONCENTRADOR } \\
1\end{array}$ & $\begin{array}{ll}\text { - } & \text { Base de dados } \\
& \text { histórica } \\
\text { - } & \text { Base de dados } \\
\text { cadastro } \\
\text { - Discos Virtuais }\end{array}$ & \\
\hline $\begin{array}{l}\text { Dell } \\
\text { R610 }\end{array}$ & $\begin{array}{l}\text { CONCENTRADOR } \\
2\end{array}$ & $\begin{array}{l}\text { Servidor de aplicação } \\
\text { redundante SCADA } \\
\text { (Mina, Britagem e } \\
\text { Concentrador) }\end{array}$ & $\begin{array}{ll}\text { - } & \text { E3 Server } \\
\text { - } & \text { EPM Interface } \\
\text { - } & \text { E3 Dominios remotes } \\
\text { - } & \text { E3 OPC Server } \\
\text { - } & \text { Driver Ge-Fanuc } \\
& \text { ethernet } \\
\text { - } & \text { Driver Allen Bradley } \\
& \text { ABFD1 } \\
\end{array}$ \\
\hline $\begin{array}{l}\text { Dell } \\
\text { R610 }\end{array}$ & $\begin{array}{l}\text { CONCENTRADOR } \\
2\end{array}$ & $\begin{array}{l}\text { Servidor de historização } \\
+ \text { BD (backup) }\end{array}$ & $\begin{array}{ll}\text { - } & \text { EPM Server } \\
\text { - } & \text { SQL Server } 2008 \\
\text { - } & \text { E3 Web Server } \\
\end{array}$ \\
\hline
\end{tabular}

\subsubsection{Virtualização}

Optou-se pela arquitetura virtualizada para restruturação dos sistemas de supervisão da Samarco. A arquitetura foi definida pela Samarco e implementada pela Dell, em conjunto com a Samarco. Dentre as vantagens apresentadas em ambientes virtualizados pode-se destacar:

- Consolidação de servidores;

- Independência de hardware subjacente;

- Aumento do tempo de obsolescência da solução;

- Balanceamento dinâmico de carga;

- Agilidade na recuperação de desastres;

- Ambiente de teste para implantação.

- Como ferramenta de virtualização foi escolhido o VMware vSphere 5 pelos seguintes motivos:

- Nível de maturidade já atingido pelo hipervisor e liderança, com $86 \%$ de participação de mercado [1];

- Compatibilidade e parcerias desenvolvidas com a maior parte dos fornecedores de softwares de automação.

* Contribuição técnica ao $18^{\circ}$ Seminário de Automação e TI Industrial, 23 a 26 de setembro de 2014, São Paulo, SP, Brasil. 


\section{AUTOMAÇÃO \& TI}



Figura 2 - Arquitetura Samarco

No que se refere ao plano de recuperação de desastres, uma estratégia de replicação de dados entre os storages da Planta 01 e 02 foi configurada, estabelecendo uma redundância do tipo cold standby. Como as máquinas virtuais estão armazenadas nos storages, a sincronia dos dados permite a partida das mesmas no site backup, nesse caso, Planta 02. No entanto, essa operação implica em pesado uso de rede, dessa forma, nem toda estrutura pôde ser sincronizada, apenas os servidores de aplicação e historização foram escolhidos para a replicação.

\subsection{Entidades do Sistema}

Os clp's são compostos de diversos elementos que tem a função de controlar todos os dispositivos a ele interligados. A entidade controlador no sistema HSP é a representação de um controlador lógico programável. Relacionadas à instância de um controlador, existem seis entidades: Analógicas, Digitais, Equipamentos, Loops de Controle, Parâmetros e Totalizadores. Associadas a cada entidade, por sua vez, existem as instâncias do controlador (Figura 3). As modificações de cadastro das entidades do sistema são realizadas por um aplicativo desenvolvido para o projeto denominado HSP (HMI Smart Process), cujo principal usuário é a equipe de automação da Samarco.

* Contribuição técnica ao $18^{\circ}$ Seminário de Automação e TI Industrial, 23 a 26 de setembro de 2014, São Paulo, SP, Brasil. 



Figura 3 - HSP - Sistema para configuração das entidades

Para cada entidade do sistema, existe uma janela para cadastro/modificação da mesma. A seguir é apresentado, como exemplo, a janela da entidade Equipamento.



Figura 4 - Detalhe do cadastro da entidade Equipamento

Esta funcionalidade permite a equipe de manutenção do sistema de automação realizar alterações nos parâmetros das entidades do sistema de forma mais ágil e segura, pelas seguintes razões. Primeiro, deixa de ser necessário se iniciar o módulo de desenvolvimento do software do sistema de supervisão para alteração de um parâmetro de uma entidade, como por exemplo, se alterar a descrição de um equipamento ou o nível de alarme de uma analógica. Segundo, torna-se possível criar novas entidades em modo on-line, inclusive com a facilidade de se criar uma nova entidade a partir de uma outra entidade já existente. Terceiro, tem-se o rastreamento de todas as inclusões/alterações realizadas nas entidades existentes.

* Contribuição técnica ao $18^{\circ}$ Seminário de Automação e TI Industrial, 23 a 26 de setembro de 2014, São Paulo, SP, Brasil. 
Por último, o sistema permite a configuração por usuário, das permissões para inclusão, modificação e/ou visualização das entidades. Além destas funcionalidades o sistema permite:

- Realizar comparações de versões das modificações realizadas nas entidades. Esta funcionalidade proporciona um controle maior sobre uma instância, permitindo identificar o que os usuários têm feito sobre cada instância;

- Rastrear a utilização de uma determinada instância do sistema (referência cruzada);

\subsubsection{Alarmes do sistema}

São considerados alarmes uma situação de anormalidade operacional da planta que exige uma resposta do operador. A efetiva gestão dos alarmes do sistema foi considerada chave para o sucesso do projeto desde sua concepção. Neste sentido, uma série de melhorias foram implementadas visando prover recursos para anunciar, registrar e visualizar os alarmes e eventos do sistema.

Mais especificamente, os alarmes cadastrados no sistema podem ser agrupados em categorias. As categorias são utilizadas para possibilitar a aplicação de filtros no sistema de supervisão. Cada alarme do sistema deve ser associado a um grau de severidade. As severidades definem uma prioridade no tratamento de alarmes no sistema de supervisão. São quatro as divisões quanto à severidade dos alarmes: "DESARME"; "ALARMES ALTOS"; "ALARMES MÉDIOS"; "ALARMES BAIXOS". Todos os alarmes que desarmam algum equipamento aparecem na aba "DESARME", todos que forem alarmes altos aparecem na aba "ALARMES ALTOS", todos que forem alarmes médios aparecem na aba "ALARMES MÉDIOS" e, por fim, todos que forem alarmes baixos aparecem na aba "ALARMES BAIXOS".

\subsubsection{Atributos}

Os alarmes possuem os seguintes atributos:

- Condição de ativação do alarme

- Prioridade

- Dados de identificação do alarme (Classe,Tag, Descrição do tag, Mensagem do alarme, Área de processo, tela de processo associada)

- Dados de contexto (Textos ação / conseqüência para operador/tempo de resposta)

- Dados de registro do alarme (data/hora, estado, operador de reconhecimento, estação de reconhecimento)

- Condição de supressão automática

- Filtro de Tempo (delay time)

- Banda Morta

- Banner de Exibição (processo, automação ou manutenção) A relevância de cada atributo depende da classe no qual o alarme pertence. As definições dos atributos de alarmes é realizada na etapa de identificação e racionalização de alarmes, conforme critérios estabelecidos no documento de filosofia de alarmes.

\subsubsection{Funcionalidades}

As funcionalidades aplicáveis aos alarmes são as seguintes:

- Reconhecimento: É um comando de reconhecimento do operador para um alarme ativo. O reconhecimento é aplicável conforme a classe do alarme. Essa ação implica em uma mudança de estado do alarme.

\footnotetext{
* Contribuição técnica ao $18^{\circ}$ Seminário de Automação e TI Industrial, 23 a 26 de setembro de 2014,
} São Paulo, SP, Brasil. 
- Registro em Log: Toda mudança de estado do alarme é persistida em banco de dados para análise histórica. Os dados históricos são consultáveis pelo usuário em tela de sumário histórico.

- Exibição em tempo real: Toda mudança de estado do alarme é exibida em tempo real pelo sistema. Os alarmes são visualizados no banner, sumário ou sinóticos conforme definição da classe de alarmes.

- Supressão automática: O alarme pode ser suprimido pelo sistema, caso uma determinada condição de supressão seja satisfeita. Nesta condição o alarme não é exibido ao operador em tempo real (banner e sumário de tempo real), apesar de ainda ser armazenado em log.

- Supressão Manual: O alarme pode ser suprimido pelo operador. Nesta condição o alarme não é exibido ao operador em tempo real (banner e sumário de tempo real), apesar de ainda ser armazenado em log. Após determinado tempo configurado o alarme retorna ao estado de não supressão.

- Navegação para tela de processo: A partir da mensagem de alarme no banner ou sumário de alarmes é possível navegar até a tela de processo relacionada à condição de disparo do alarme.

\subsubsection{Estados}

O sistema distingue os seguintes estados de alarmes:

- Normal: É dito normal quando condição de disparo do alarme não está mais ativa e não há pendência de reconhecimento pelo operador.

- Ativo não-reconhecido: É dito Ativo não-reconhecido quando a condição de disparo de alarme é satisfeita e há pendência de reconhecimento pelo operador.

- Ativo reconhecido: É dito Ativo reconhecido quando a condição de disparo de alarme ainda é satisfeita e não há pendência de reconhecimento pelo operador

- Inativo Não reconhecido: É dito Inativo não-reconhecido quando a condição de disparo de alarme não é mais satisfeita e há pendência de reconhecimento pelo operador.

- Suprimido automaticamente: É dito suprimido automaticamente quando a condição de supressão automática é satisfeita.

\subsubsection{Classes de alarmes}

As classes são agrupamentos de alarme que reúnem uma série de atributos, estados e tratamentos semelhantes. Este sistema considera as seguintes classes:

- Alarmes de processo: sinais que representam uma condição insegura ou de anormalidade no processo e que necessitam de uma ação do operador. Esta classe se subdivide nas seguintes classes:

- Alarmes de defeito de equipamentos: Esta classe representa todos os alarmes derivados dos defeitos de equipamentos. Somente os defeitos que efetivamente pararam um equipamento são exibidos ao operador.

- Alarmes de intertravamentos de equipamentos: Esta classe representa os alarmes derivados dos intertravamentos de equipamentos, caso 0 intertravamento seja cadastrado como gerador de alarme.

- Alarmes de limites de analógica: Esta classe representa todos os alarmes derivados dos limites de analógica $(\mathrm{HH}, \mathrm{H}, \mathrm{L}$ e LL).

\footnotetext{
* Contribuição técnica ao $18^{\circ}$ Seminário de Automação e TI Industrial, 23 a 26 de setembro de 2014,
} São Paulo, SP, Brasil. 
- Alarmes de desvio de PID: Esta classe representa os alarmes de desvio de SP das malhas de controle.

- Alarmes de digitais: Esta classe representa os alarmes derivados das digitais cadastradas como geradores de alarmes.

- Alarmes de sistema: sinais que representam uma condição anormal do sistema de supervisão ou de qualquer dispositivo que compõe a arquitetura de automação. Esta classe se subdivide nas seguintes classes:

- Alarmes de Automação: Esta classe representa os alarmes relacionados aos ativos de responsabilidade da Automação

- Alarmes de Manutenção: Esta classe representa os alarmes relacionados aos ativos de responsabilidade da manutenção.

A seguir é apresentado uma tabela que resume os atributos, estados e funcionalidades aplicáveis a cada classe de alarme.

Tabela 2. Resumo dos atributos, estados e funcionalidades as classes de alarme

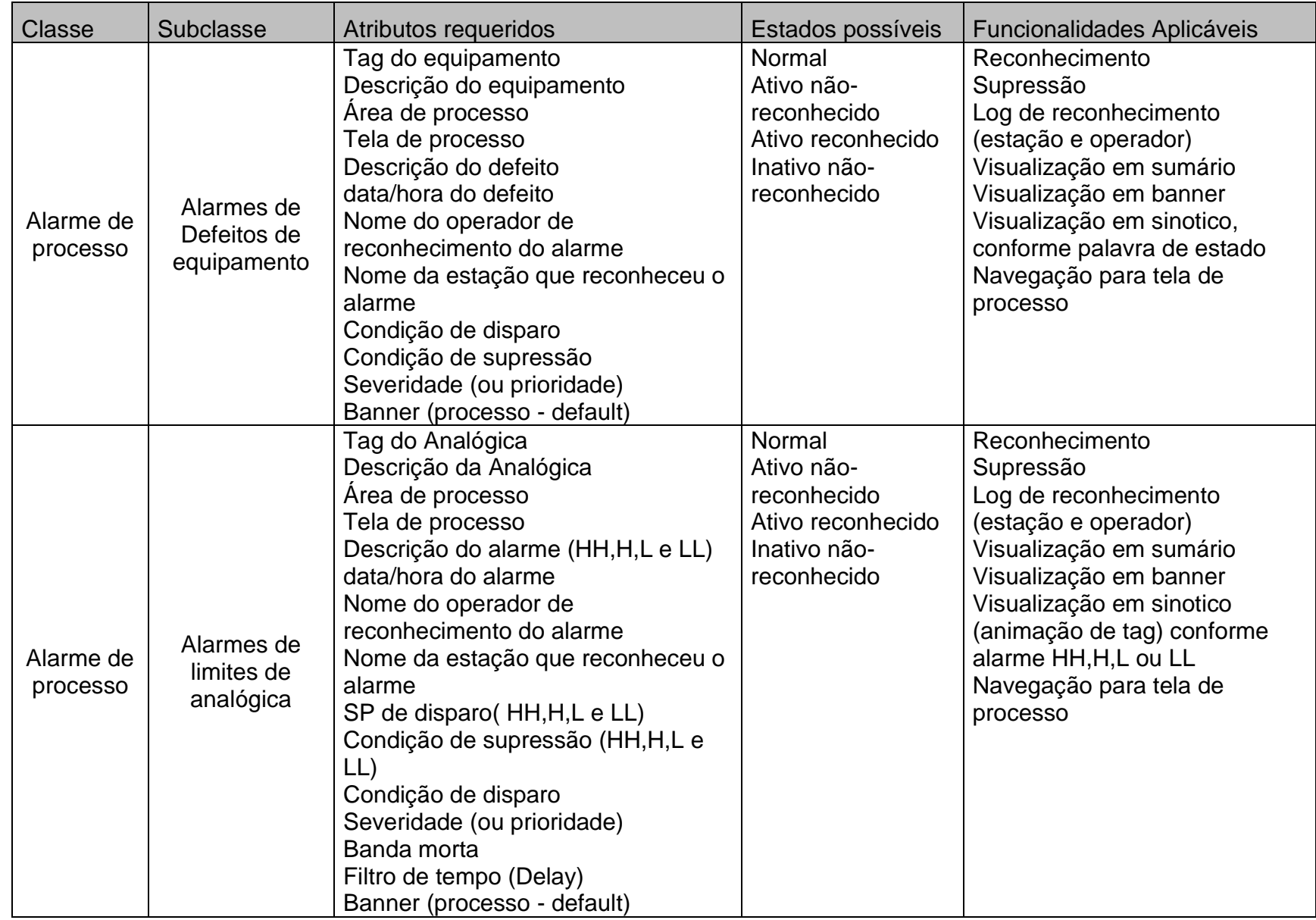

* Contribuição técnica ao $18^{\circ}$ Seminário de Automação e TI Industrial, 23 a 26 de setembro de 2014, São Paulo, SP, Brasil. 
AUTOMAÇÃO \& TI

\begin{tabular}{|c|c|c|c|c|}
\hline $\begin{array}{l}\text { Alarme de } \\
\text { processo }\end{array}$ & $\begin{array}{c}\text { Alarmes de } \\
\text { desvio de PID }\end{array}$ & $\begin{array}{l}\text { Tag do PID } \\
\text { Descrição da PID } \\
\text { Área de processo } \\
\text { Tela de processo } \\
\text { Descrição do alarme (HH,H,L e LL) } \\
\text { data/hora do alarme } \\
\text { Nome do operador de } \\
\text { reconhecimento do alarme } \\
\text { Nome da estação que reconheceu o } \\
\text { alarme } \\
\text { SP de disparo( HH,H,L e LL) } \\
\text { Condição de supressão (HH,H,L e } \\
\text { LL) } \\
\text { Condição de disparo } \\
\text { Sevidade (ou prioridade) } \\
\text { Banda morta } \\
\text { Filtro de tempo (Delay) } \\
\text { Banner (processo - default) }\end{array}$ & $\begin{array}{l}\text { Normal } \\
\text { Ativo não- } \\
\text { reconhecido } \\
\text { Ativo reconhecido } \\
\text { Inativo não- } \\
\text { reconhecido }\end{array}$ & $\begin{array}{l}\text { Reconhecimento } \\
\text { Supressão } \\
\text { Log de reconhecimento } \\
\text { (estação e operador) } \\
\text { Visualização em sumário } \\
\text { Visualização em banner } \\
\text { Visualização em sinotico } \\
\text { (animação de tag) conforme } \\
\text { alarme HH,H,L ou LL } \\
\text { Navegação para tela de } \\
\text { processo }\end{array}$ \\
\hline $\begin{array}{l}\text { Alarme de } \\
\text { processo }\end{array}$ & $\begin{array}{l}\text { Alames de } \\
\text { digitais }\end{array}$ & $\begin{array}{l}\text { Tag da digital } \\
\text { Descrição da digital } \\
\text { Área de processo } \\
\text { Tela de processo } \\
\text { Descrição da digital } \\
\text { data/hora do alarme } \\
\text { Nome do operador de } \\
\text { reconhecimento do alarme } \\
\text { Nome da estação que reconheceu o } \\
\text { alarme } \\
\text { Condição de supressão } \\
\text { Condição do disparo } \\
\text { Severidade (ou prioridade) } \\
\text { Banner (processo - default) }\end{array}$ & $\begin{array}{l}\text { Normal } \\
\text { Ativo não- } \\
\text { reconhecido } \\
\text { Ativo reconhecido } \\
\text { Inativo não- } \\
\text { reconhecido }\end{array}$ & \begin{tabular}{|l} 
Reconhecimento \\
Supressão \\
Log de reconhecimento \\
(estação e operador) \\
Visualização em sumário \\
Visualização em banner \\
Visualização em sinotico \\
conforme palavra de digital \\
(quando necessário) \\
Navegação para tela de \\
processo
\end{tabular} \\
\hline $\begin{array}{l}\text { Alarme de } \\
\text { sistema }\end{array}$ & $\begin{array}{l}\text { Alarmes de } \\
\text { automação }\end{array}$ & $\begin{array}{l}\text { Tag do equipamento } \\
\text { Descrição do equipamento } \\
\text { Área de processo } \\
\text { Tela de processo } \\
\text { Descrição do defeito } \\
\text { data/hora do defeito } \\
\text { Nome do operador de } \\
\text { reconhecimento do alarme } \\
\text { Nome da estação que reconheceu o } \\
\text { alarme } \\
\text { Condição de disparo } \\
\text { Condição de supressão } \\
\text { Severidade (ou prioridade) } \\
\text { Banner (Automação - default) }\end{array}$ & $\begin{array}{l}\text { Normal } \\
\text { Ativo não- } \\
\text { reconhecido } \\
\text { Ativo reconhecido } \\
\text { Inativo não- } \\
\text { reconhecido }\end{array}$ & \begin{tabular}{|l|} 
Reconhecimento \\
Supressão \\
Log de reconhecimento \\
(estação e operador) \\
Visualização em sumário \\
Visualização em banner \\
Visualização em sinotico \\
conforme palavra de digital \\
(tela arquitetura) \\
Navegação para tela de \\
processo
\end{tabular} \\
\hline $\begin{array}{l}\text { Alarme de } \\
\text { sistema }\end{array}$ & $\begin{array}{l}\text { Alarmes de } \\
\text { Manutenção }\end{array}$ & $\begin{array}{l}\text { Tag do equipamento } \\
\text { Descrição do equipamento } \\
\text { Área de processo } \\
\text { Tela de processo } \\
\text { Descrição do defeito } \\
\text { data/hora do defeito } \\
\text { Nome do operador de } \\
\text { reconhecimento do alarme } \\
\text { Nome da estação que reconheceu o } \\
\text { alarme } \\
\text { Condição de disparo } \\
\text { Condição de supressão } \\
\text { Severidade (ou prioridade) } \\
\text { Banner (Manutenção - default) }\end{array}$ & $\begin{array}{l}\text { Normal } \\
\text { Ativo não- } \\
\text { reconhecido } \\
\text { Ativo reconhecido } \\
\text { Inativo não- } \\
\text { reconhecido }\end{array}$ & $\begin{array}{l}\text { Reconhecimento } \\
\text { Supressão } \\
\text { Log de reconhecimento } \\
\text { (estação e operador) } \\
\text { Visualização em sumário } \\
\text { Visualização em banner } \\
\text { Visualização em sinotico } \\
\text { conforme palavra de digital } \\
\text { (tela arquitetura) } \\
\text { Navegação para tela de } \\
\text { processo }\end{array}$ \\
\hline
\end{tabular}




\begin{tabular}{|l|l|l|} 
Tag do equipamento & Normal & Reconhecimento \\
Descrição do equipamento & Ativo não- & Supressão \\
Área de processo & reconhecido & Log de reconhecimento \\
Tela de processo & Ativo reconhecido & (estação e operador) \\
Descrição do defeito & Inativo não- & Visualização em sumário \\
data/hora do defeito & Visualizçãão em banner \\
Nome do operador de & reconhecido & Visualizção em sinotico \\
reconhecimento do alarme & & conforme palavra de digital \\
Nome da estação que reconheceu & & (tela arquitetura) \\
o alarme & Navegação para tela de \\
Condição de disparo & processo \\
Condição de supressão & \\
Severidade (ou prioridade) & & \\
Banner (Manutenção - default) & &
\end{tabular}

\section{CONCLUSÃo}

O projeto de modernização do sistema de automação e controle da Mina, Britagem e Concentrador I da Samarco mineração disponibiliza a equipe de operação e manutenção, uma série de ferramentas que possibilitam um alto nível de monitoração e flexibilidade a equipe de operação da planta e manutenção do sistema de automação. A tolerância à falha e o consequente aumento da disponibilidade, sobretudo no que tange a recuperação de desastres, é um dos benefícios obtidos com virtualização. Destaca-se ainda neste projeto a preocupação por uma solução tecnológica abrangente no que se diz respeito a todas as funcionalidades solicitadas pela Samarco.

\section{Agradecimentos}

Agradecemos a todos os envolvidos na fases de especificação e desenvolvimento do projeto. Em especial, as equipes de campo da Samarco e da IHM Engenharia, que não mediram esforços para que as atividades fossem realizadas com sucesso durantes as paradas programadas.

\section{REFERÊNCIAS}

1 lqbal MS, Molloy CM, Rymarczyk J. IT Virtualization Best Practices. Mc Press; 1st Edition (January, 2011)

* Contribuição técnica ao $18^{\circ}$ Seminário de Automação e TI Industrial, 23 a 26 de setembro de 2014, São Paulo, SP, Brasil. 